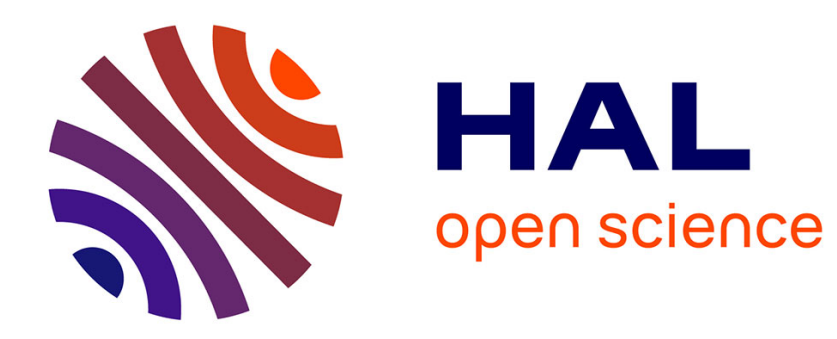

\title{
Editorial. Encrer les eaux courantes : la géographie prise au mot
}

Yves-François Le Lay

\section{To cite this version:}

Yves-François Le Lay. Editorial. Encrer les eaux courantes: la géographie prise au mot. Géocarrefour

- Revue de géographie de Lyon, 2013, 88 (1), pp.3-13. 10.4000/geocarrefour.8913 . halshs-00862430

\section{HAL Id: halshs-00862430 \\ https://shs.hal.science/halshs-00862430}

Submitted on 16 Sep 2013

HAL is a multi-disciplinary open access archive for the deposit and dissemination of scientific research documents, whether they are published or not. The documents may come from teaching and research institutions in France or abroad, or from public or private research centers.
L'archive ouverte pluridisciplinaire HAL, est destinée au dépôt et à la diffusion de documents scientifiques de niveau recherche, publiés ou non, émanant des établissements d'enseignement et de recherche français ou étrangers, des laboratoires publics ou privés. 
Le Lay Y.-F., 2013. « Editorial. Encrer les eaux courantes : la géographie prise au mot ». Géocarrefour, 88, 1, p. 3-13.

\section{Editorial. Encrer les eaux courantes : la géographie prise au mot}

Rapprocher discours et cours d'eau ne doit pas étonner: leur étymologie elle-même les rassemble. Fondé sur le substantif "cours" qui désigne l'action de courir, le mot discours provient de discursus et a d'abord signifié en latin classique l'action de courir çà et là, à différents endroits - du fait de son préfixe marquant la distinction-, puis discours, conversation ou entretien en bas-latin. Dès le Moyen Age, le cours se référait aux hommes, aux bateaux, aux sentiments, au temps, mais aussi au mouvement d'une eau courante. De même, en anglais, discourse et watercourse partagent le même radical.

Le cours de la parole et celui de l'eau ont plusieurs points communs. La parole s'écoule et ses flots peuvent indisposer; l'eau murmure et parle au contemplatif. Le flux de l'eau comme celui des mots occupent l'espace et s'y diffusent. Ils évoluent avec le temps : le débit d'une rivière ou d'une parole varie selon les heures de la journée, les saisons ou les années. Le discours et le cours d'eau sont ainsi marqués par une dimension principale, diachronique ou chronologique, qui domine tellement la perception que les autres dimensions ont longtemps été négligées : les cours de l'eau et des mots étant orientés, tout deux présentent un début et une fin. Si le discours correspond à une séquence ordonnée de phrases employées concrètement pour transmettre des informations, le cours d'eau s'écoule d'amont en aval depuis sa source jusqu'à sa partie terminale. Ainsi le torrent a-t-il son bassin de réception, son chenal d'écoulement et son cône de déjections. D'une façon semblable à l'auteur d'un discours argumenté qui s'efforce d'enchaîner une introduction, un développement structuré et une conclusion, les écologues ont porté beaucoup d'attention à cette dimension longitudinale des cours d'eau : "Des têtes de bassin à l'embouchure, un réseau fluvial offre un gradient continu de conditions physiques" (Vannote et al., 1980, p. 130). Puis la pluralité des dimensions (longitudinale, latérale et verticale) et des échelles spatiales et temporelles a été reconnue au cours des années 1980 (Frissell et al., 1986 ; Ward, 1989). De même, un texte gagne à être étudié d'après des échelles distinctes, tantôt infra-textuelle - celles des caractères, des mots, des phrases, des paragraphes - tantôt supra-textuelles ou contextuelles, à la faveur des notions d'intertextualité ou de situation d'énonciation par exemple. En effet, l'approche systémique, qui a notamment emprunté au structuralisme et à la théorie de l'information, s'est diffusée dans toutes les disciplines, y compris la linguistique (Durand, 2010) ; les objets d'étude sont désormais considérés comme des systèmes ouverts sur leur environnement avec lequel ils entretiennent des relations.

Le discours et l'eau courante servent de fils directeurs à ce numéro. En effet, les eaux courantes suscitent des discours oraux et écrits que les ruptures et crises, en particulier, alimentent et renouvellent. Ces discours sont une fenêtre ouverte sur les activités, les représentations et la gestion du fleuve. Il est au moins trois notions - l'attitude, le risque et le paysage - susceptibles de mettre en cohérence ces éléments. Elles font le lien entre d'une part l'évaluation que les hommes réalisent à l'égard de leur environnement et d'autre part la manière dont ils s'y comportent, ce qu'ils en disent ou ce qu'ils y font. Le risque et l'angoisse qui lui est associée nous poussent à agir. Or, chercher à "prendre au mot" la géographie sociale et culturelle de l'environnement, c'est ainsi estimer que le discours constitue précisément une voie d'accès vers les attitudes environnementales. A la faveur d'un tournant linguistique et discursif, c'est considérer que ce qui est dit et écrit médiatise une part de la réalité et y donne une prise.

\section{L'angoisse d'habiter la Terre : le risque comme agent producteur du paysage}


Pourquoi appréhender les mots? Ils intéressent moins en eux-mêmes que pour ce qu'ils révèlent des motivations à intervenir sur ce qui nous entoure : ils disent quelque chose des interactions entre les individus et leur environnement, donnant ainsi un peu d'épaisseur au paradigme de l'interface hommes-nature (Figure 1).

Le comportement, l'acte et le discours: des fenêtres sur les représentations de l'environnement

Le comportement est défini comme une réalité observable, sous la forme d'actes - y compris l'écriture ou la parole - qui soulignent les interactions entre un sujet (en l'occurrence un énonciateur) et son environnement (qui peut être réduit à une situation d'énonciation). Cet individu présente plusieurs niveaux de réalité : (a) un niveau biologique, parce qu'il est muni d'un organisme neurophysiologique, avec ses besoins et ses exigences, (b) un niveau anthropologique, étant donné qu'une personnalité l'individualise, en particulier son affectivité et son activité cognitive, (c) un niveau social, dans la mesure où il se trouve en interaction avec d'autres acteurs, et (d) un niveau culturel, qui est celui des normes, des modèles, des valeurs et des idéologies. Tout acte s'inscrit dans ces quatre contextes à la fois ; il résulte toujours d'une interaction d'influences provenant de chacun d'eux.

Dans les années 1980, A. Berque publia une série d'articles dans L'Espace géographique où il développa sa notion de trajection (Berque, 1984, 1985 et 1987). Née de la problématique de la combinaison du subjectif et de l'objectif, elle alimente une approche intégrée des faits trajectifs - comme les paysages, les risques et les nuisances - qui combinent l'en-soi des choses et le regard des hommes, l'intérêt qu'ils leur portent. Le point de vue adopté est celui de la géographie culturelle, entendue comme "l’étude du sens des milieux" (Berque, 1987, p. 242), c'est-à-dire le sens global et unitaire qu'"une société donne à sa relation à l'espace et à la nature ; relation que le paysage exprime concrètement" (Berque, 1984, p. 33). Pour employer un autre néologisme de l'auteur (1990), cette géographie culturelle étudie les médiances. Le paysage existe dans sa relation à un sujet collectif - un groupe social muni d'une histoire. Il peut être considéré comme la (re)production d'une logique qu'il importe de décrypter. Ainsi le paysage fluvial est-il une empreinte en ceci qu'il exprime une civilisation et une matrice dans la mesure où il oriente le regard et le comportement des hommes (Berque, 1984). La relation médiale affirme son ambivalence parce qu'elle tient du social et du naturel, du collectif et de l'individuel, du subjectif et de l'objectif. A. Berque penche néanmoins du côté social ; le sujet engagé dans la relation médiale est collectif : "ce qui correspond à un milieu, c’est une société" (Berque, 1987, p. 242).

Or ne serait-il pas possible de prendre le parti de placer l'individu au cœur de la réflexion ? De fait, ce sont bien des individus qui perçoivent et modifient l'environnement. Selon R. Brunet (1974, p. 191), "tous les travaux parlent complaisamment de l'homme, au singulier et si possible avec un grand $\mathrm{H}$. Mais quelle est donc cette abstraction ? L'Homme n'existe pas : qui l'a rencontré ? Les hommes existent". Reconnaître cet abus permet de travailler à diverses échelles actorielles - celles de l'individu, du groupe ou encore de la société - et de montrer la pluralité des attitudes. Chaque individu perçoit le réel et en construit des images en effectuant un tri d'informations, une perception sélective des éléments qui l'environnent. La vision du monde est strictement personnelle : toute personne construit sa propre Umwelt, le monde de perception et d'action qui lui est spécifique. Le système de pertinence d'un individu est fonction de l'ensemble des problèmes spécifiques qui le préoccupent et des projets qu'il a au moment où il considère ce monde. Brunet (1974) explique ainsi le décalage entre réel et image du réel par l'existence d'un filtre perceptif. Cette image du réel peut être mémorisée et l'on devine déjà quel rôle peut jouer l'expérience vécue, c'est-à-dire l'image d'une 
catastrophe lors de la construction du risque. Mais elle peut aussi être imaginée et, dans ce cas, le poids de l'ensemble de l'information engrangée apparaît comme déterminant lors de la conception de l'image imaginée. Or, les mots du discours permettent de communiquer ces images, qu'elles soient mémorisées ou imaginées.

Mémorisation, évaluation, anticipation... Autant de capacités qui influencent nos attitudes et nos discours à caractère environnemental et dont a cherché à rendre compte le modèle heuristique du cerveau triunique (Figure 1), revisité par Morin (1994). Ce dernier distingue en effet trois cerveaux qui prennent en charge ces différentes compétences (MacLean, 1990 ; Durand, 2010). Le paléo-cerveau assure la survie de l'organisme en contrôlant les fonctions vitales et influence l'essentiel du comportement automatique et instinctif, comme le sens de l'orientation et les gestes défensifs. Le système limbique permet de mémoriser les comportements agréables et désagréables; il joue un rôle majeur dans l'expression des émotions si bien qu'il a été considéré comme le siège des jugements de valeur. Enfin, le néocortex sert de fondement au langage, à la pensée abstraite, à l'imagination, à l'anticipation et à la conscience ; il dote les hommes d'une capacité d'apprentissage et leur permet de poser des problèmes et de les résoudre. Tant par sa structure que par son fonctionnement, un tel modèle se montre ainsi propice à l'émergence d'une tension entre l'émotion et la raison (Koestler, 1979), entre homo sapiens et homo demens : "l'homme de la rationalité est aussi celui de l'affectivité du mythe et du délire" (Morin, 2000, p. 62).

\section{Explorer les attitudes à l'égard de l'environnement}

Comment expliquer cette motivation qui nous pousse à agir sur notre environnement ? Un psychologue américain, Heider (1946), a postulé que chaque individu se situe dans un "champ de forces" et qu'il recherche l'ordre, la symétrie et la cohérence entre les éléments de son environnement. Si une contradiction apparaît, sa préférence pour un monde équilibré peut le conduire à modifier sa conception ou sa connaissance des choses. Festinger (1957) a creusé l'idée et développé une théorie de la dissonance cognitive, utilisée ensuite dans le cadre de la géographie des risques, par exemple par Schoeneich et Busset-Henchoz (1998). Chez Heider, le sujet est davantage un analyste ou un observateur qu'un acteur ; alors que Festinger estime que l'état d'inconsistance est induit par le comportement du sujet lui-même. Ce dernier dispose de cognitions - c'est-à-dire des connaissances, des opinions ou des croyances - sur lui-même et sur son environnement. Si ses cognitions ne sont pas compatibles avec son comportement, le sujet ressent un état de tension, dit "état de dissonance cognitive". Son axe corticotrope est alors sollicité, ce qui libère le neuromédiateur du stress (Laborit, 1994) et motive l'individu à changer quelque chose pour retrouver une consistance entre attitude et comportement.

Le concept de représentation est très utilisé dans le champ des Sciences humaines et sociales (SHS), y compris en géographie (Roussiau et Bonardi, 2001). Selon Flament (1994, p. 37), "on peut dire qu'une représentation sociale est un ensemble organisé de cognitions relatives à un objet, partagées par les membres d'une population homogène par rapport à cet objet". Bien que la frontière ne soit pas nette entre la représentation et l'attitude, cette dernière a néanmoins l'intérêt d'être mieux structurée et de déboucher sur les questions de pratiques et d'aménagement. Avec le concept d'attitude (Ajzen, 2005), la psychologie sociale a proposé un cadre conceptuel commode pour appréhender l'interface hommes-nature : il désigne une structure mentale abstraite qui sert d'intermédiaire entre les objets attitudinaux (tout objet de nature, y compris la rivière) et les réponses individuelles (comme les demandes d'entretien ou de restauration des cours d'eau). C'est pourquoi il a été mobilisé par quelques géographes (par exemple Gold, 1980 ; Golledge et Stimson, 1997 ; Kah, 2000 ; Depraz, 2005). Dans la mesure où il n'existe pas d'attitude sans objet d'attitude dans une situation sociohistorique, les 
géographes peuvent y réinvestir leur intérêt pour l'espace, le temps, les acteurs et les systèmes. Selon Triandis (1971, p. 2), une "attitude est une idée chargée d'émotion qui prédispose une catégorie d'actions à une catégorie particulière de situations sociales". Une telle définition débouche sur le modèle classique de l'attitude (Figure 2) dont l'organisation reconnaît trois composantes dites cognitive, affective et conative (Vallerand, 2006). La dimension cognitive renvoie aux croyances ou opinions qu'évoque un objet d'attitude. Les cognitions participent à la construction et l'interprétation de la réalité ; elles sont signifiantes, tantôt figuratives/imaginantes, tantôt verbales/linguistiques. La dimension affective est associée à l'émotion suscitée et consiste en la réponse évaluative - favorable ou défavorable à l'objet d'attitude. C'est en cela qu'elle s'avère motivante. La dimension conative concerne l'anticipation de l'action ; elle oriente les conduites et comportements.

Les textes et les paroles permettent-ils d'inférer les attitudes tenues à l'égard d'un objet ? Oui. Si des données non verbales peuvent être mesurées et interprétées - des observations perceptuelles, physiologiques et comportementales disent respectivement quelque chose de la cognition, de l'affect et de la conation -, les trois composantes de l'attitude peuvent également être appréhendées par des déclarations verbales (Rosenberg et al., 1960). Celles-là rendent compte des éléments d'opinion, des affects et des intentions d'action (Figure 2).

\section{Du risque à l'action environnementale}

Les discours tenus sur l'eau courante sont ambivalents. Derrière l'eau pure et revigorante se cache l'eau qui enlève ce à quoi les sociétés tiennent le plus. Les gestionnaires des milieux aquatiques n'oublient pas la vulnérabilité des enjeux et les risques qui leur sont associés. Quand il s'agit de risque, les mots sont polysémiques (Figure 3). Quelques jalons peuvent être posés (Thouret et d'Ercole, 1996 ; Pigeon, 2005 ; Veyret et Reghezza, 2006). D'une part, un danger désigne une entité ou un processus pourvu d'une propension à générer des dommages - appelée dangerosité -, par exemple le débordement d'un cours d'eau. Le terme "menace" gagne à être écarté du fait de son ambiguïté : il peut concerner le signe par lequel se manifeste ce qu'on doit craindre et non l'objet même de cette crainte. Plus particulièrement, l'aléa reste est une modalité à temporalité ponctuelle du danger. Si le danger peut être structurel ou conjoncturel, l'aléa est étymologiquement conjoncturel. D'autre part, un enjeu désigne une entité positivement valorisée et pourvue d'une propension à subir des dommages. Par conséquent, il y a catastrophe lorsque l'inscription spatio-temporelle du danger et de l'enjeu est au moins en partie commune, autrement dit quand, au même endroit et au même moment, on a un danger et un enjeu. Si le risque est dit (maladroitement) naturel, technologique ou social, il procède toujours d'une production humaine : c'est l'anticipation (anxiogène) de la catastrophe. La notion de risque renvoie à celle d'attitude. Il procède de l'anticipation de l'occurrence imprévisible, mais vraisemblable, d'une situation crainte (dite catastrophe, crise ou accident). Cette incertitude est inhérente au risque ; elle découle d'un déficit informationnel : s’il y a certitude, il n’y a plus de risque. De plus, le risque est associé à un comportement contre-attitudinel, et plus précisément contre-sécuritaire : la manière d'être ou d'agir de l'individu n'est pas en accord avec une valeur largement partagée, la sécurité. Que de formulations ambiguës ! De fait, il n’y a pas de "risque naturel" puisqu'il n’est de risque que représenté et vécu. De même, la "perception du risque" est aberrante et c'est en ceci que les termes "catastrophe" et "risque" méritent d'être distingués: si la catastrophe peut être perçue, vécue et représentée, le risque est une représentation (anticipation) de la catastrophe. Pour reprendre les éléments du cerveau triunique, le risque est un modèle intériorisé que le néocortex du référentiel construit. Son rôle est de conserver de l'information, sous une forme utilisable, pour des objectifs comportementaux ultérieurs, dans la perspective d'une meilleure adaptation à l'environnement. Concrètement, un riverain peut 
anticiper une inondation après avoir acquis une maison positionnée dans le lit majeur d'un cours d'eau; il anticipe alors l'endommagement d'un bien auquel il tient. L'activité intériorisée de son système nerveux active le système limbique qui donne une couleur affective (ou valence) à l'information, sur la base d'un système de valeurs (au sens de principes ou aspirations). Le plus souvent, quand on anticipe une inondation, la valence est plutôt négative; c'est désagréable. De fait, la valorisation du risque est généralement dysphorique. La motivation accompagne la "prise de conscience du risque". Cette dernière expression est commode mais malheureuse puisqu'un risque ne peut être sans reconnaissance : il n'existe pas de risque dont un individu n'ait pas conscience.

La motivation traduit la quantité d'énergie nécessaire pour augmenter l’ordre dans les structures environnementale (milieu extérieur) et psychophysiologique (milieu intérieur). Le risque est motivant et enjoint le cortex à préparer une stratégie compensatrice visant à rétablir un équilibre perturbé. Comment l'individu s'y prend-il pour résoudre sa dissonance cognitive ? Les figures 1 et 4 proposent quatre types de réponse. La fuite ou la lutte fournit une solution rapide (réponse 1 ). Il s'agit de réactions à la peur ressentie devant un danger physique (Diel, 1956). Mais l'angoisse, inhérente au risque, invite à diverses stratégies de restauration de l'équilibre cognitif. Selon les connaissances, les savoir-faire, les moyens techniques et les ressources financières, trois options sont envisageables: l'acceptance, l'adaptation ou la dominance. L'acceptance (réponse 2) désigne le fait de modifier sa structure cognitive, en ajoutant de nouvelles cognitions ou bien en altérant la charge affective qui leur est attribuée. Cette option donne l'impression d'accepter son environnement tel qu'il est ; lorsque l'action est difficile, coûteuse ou impossible, elle est privilégiée. L’illusion de contrôle et l'optimisme irréaliste conduisent les individus à penser qu'ils sont quasiment invulnérables et qu'ils disposent de plus de contrôle sur la situation qu'ils n'en ont effectivement. L'illusion de contrôle correspond à une tendance générale qui consiste à croire que l'on a plus de contrôle qu'il ne faudrait, face à des événements dont les caractères et l'occurrence sont empreints d'incertitudes. D'autre part, l'optimisme irréaliste peut être défini comme une tendance des individus à croire qu'ils seront confrontés à plus d'événements positifs et à moins d'événements négatifs que les autres personnes. Ainsi, quand nous subissons une dissonance cognitive, nous pouvons rechercher ou inventer des groupes de comparaisons plus vulnérables que nous, ceci pour réduire notre anxiété. La gestion sociale de l'information environnementale trouve ici toute son importance. Une plaquette, des articles de journaux, des panneaux de signalisation routière favorisent le risque et forcent les individus à réévaluer la connaissance qu'ils ont de leur comportement. L'individu peut également modifier son comportement (réponse 3), c'est-à-dire déménager ou abandonner une pratique : en quelque sorte, il s'adapte à son nouvel environnement. Mais les sujets tendent à privilégier la modification de leur environnement (réponse 4). C’est l'option prométhéenne. Il s'agit de réduire les pertes par une accommodation technique, pour modifier la fréquence d'occurrence de l'aléa (prévention) ou pour diminuer les dommages (protection).

$\mathrm{Au}$ total, cette réflexion conduit à nuancer la distinction souvent opérée entre ressources, contraintes et risques. Le risque apparaît comme une modalité mentale de la contrainte. Il pousse à une action intentionnelle. C'est en ceci qu'il est une bonne chose : "(...) dans le danger croît / Ce qui sauve aussi" (Hölderlin, 2005, p. 824-825). Alors que la contrainte physique consiste en une force empêchant d'agir ou faisant agir contre sa volonté, le risque est certes un déterminant, mais il est moins une cause qu'une motivation. Mieux qu'une contrainte, c'est une astreinte ou une obligation : le risque détermine au sens volitif et implique la liberté dans la mesure où seule la conscience impose.

\section{La pluralité des approches géo-discursives}


Le chercheur en SHS s'intéresse aux dits et aux écrits qui révèlent des éléments de connaissances acquises par un énonciateur, aux énoncés évaluatifs tenus à l'égard d'idées, d'objets, de situations ou de personnes, aux discours rationnels sur des actes et aux discours rationnalisant sur des actions. A quoi accède-t-il à travers un corpus textuel ? Tournier (1980, 1981 et 1993) a défendu l'idée selon laquelle il n'est pas de science sans simplification ou, pour reprendre ses termes, sans réduction dont il a distingué trois types, à savoir linguistique, heuristique et lexicométrique (Figure 5). La réduction linguistique reconnaît que la langue, prise comme réserve abstraite des virtualités, reste inaccessible. Peu importe d'ailleurs pour les SHS : trop distante de la réalité, la langue ne les intéresse que de très loin. En revanche, un corpus bien construit procède de l'échantillonnage représentatif des sources textuelles disponibles. Ces sources témoignent du discours qui a réalisé des usages caractéristiques d'une situation (dite d'énonciation, de communication, ou encore sociohistorique). Le chercheur travaille sur des actualisations d'un consensus cristallisé par un usage. Il s'efforce de voir au-delà du mur du langage; il interprète les (non) emplois de mots. La réduction heuristique est tout aussi intéressante. "Tout chercheur est d'abord un curieux. Mais la réalité qu'il rencontre dans sa curiosité est quelque chose de complexe qui a pour principal caractère d'être hétérogène et soumis au temps" (Tournier, 1993, p. 22). Il n'y a pas d'autre choix que de simplifier le réel en le réduisant à une situation d'énonciation. Celle-là est construite en posant un centre d'intérêt thématique, un cadrage spatio-temporel et un système d'hypothèses. Une telle situation d'énonciation requiert la définition d'invariants. "Le temps fluctue, fixons le temps, l'espace est diffus, bornons l'espace, les émetteurs sont foisonnants et divers, clarifions et limitons les émetteurs. Les événements langagiers sont multiformes, choisissons-les" (Tournier, 1993, p. 27). Si des variables doivent être minorisées et stabilisées, d'autres restent fluctuantes. Parmi ces variables d'étude, quelques-unes reviennent souvent dans les publications des SHS : le temps, l'espace et les acteurs. Elles facilitent la partition du corpus selon leurs modalités et conduisent ainsi à mettre à l'épreuve l'hypothèse d'une variation discursive d'une partie à l'autre du corpus, au moyen d'une approche contrastive. Par exemple, le chercheur peut opter pour un protocole qui lui permet de vérifier si le contenu des discours diverge selon qu'ils ont été produit à l'amont ou à l'aval d'un bassin versant, dans des communes riveraines de cours d'eau différents, ou encore par des catégories d'acteurs distinctes. De même, une analyse diachronique menée sur un corpus de textes publiés au cours de plusieurs années s'avère propice au repérage d'éventuelles permanences et mutations lexicales d'une année à l'autre ou d'une période à l'autre. Le travail du chercheur consiste ensuite à valider les hypothèses énonciatives par une suite d'inférences interprétatives qui remontent des constats statistiques aux usages discursifs et linguistiques dont ils sont la trace, puis aux données socio-historiques qui en constituent les causes. "L’interprétation est une mise en relation des paramètres sociolinguistiques, réduits aux quelques variables qui commandent le corpus enregistrés, et des comptages et jugements obtenus sur les listings des sorties-machine. Nous inférons des seconds vers les premiers, en transformant les constats en opinions. Tout le risque est là, à l'entrée dans la réduction des paramètres touffus de la situation en fonction d'une question de recherche et, à la sortie, dans le transfert interprétatif final. L’interprétation n'est qu'une remontée épurée de l'énonciation" (Tournier, 1980, p. 200).

\section{Questionnements thématiques}

A la manière du paysage, le discours fluvial fonctionne tout à la fois comme une empreinte et comme une matrice des attitudes tenues à l'égard des eaux courantes: les entretiens retranscrits, les réponses aux questions ouvertes et les différents écrits servent de réceptacles aux connaissances communes et aux acquis scientifiques, mais ils sont aussi susceptibles 
d'orienter les usages et les pratiques de la rivière. Ces discours diffusent les décisions politiques et les événements marquants de la vie sociale. De fait, les récits se sont avérés propices à l'analyse des événements hydrologiques paroxystiques (Allard, 2005), des conflits d'usages et de voisinage en milieu rural (Caron et Torre, 2006), ou encore des services environnementaux (Vicard et al., 2005). Des questions demeurent. Dans quelle mesure le discours fluvial constitue-t-il un matériau pertinent pour les analyses en SHS ? Comment ses caractères partiaux et partiels peuvent-ils renseigner sur un système fluvial ? Quels comportements (confiance, prudence, méfiance...) le chercheur doit-il adopter à son égard ?

Le discours sur les eaux courantes est pluriel ; il varie d'un acteur à l'autre selon des valeurs, des intérêts, des pratiques... En tant qu'espace multifonctionnel, si la rivière est à la fois lieu de production, de protection et de récréation, elle est aussi source d'inquiétudes. C'est pourquoi il importe non seulement de décrire le jeu des acteurs déterminants dans le processus de gestion du cours d'eau, de présenter la structure du schéma informatif, mais aussi d'évaluer comment les crises et ruptures environnementales perturbent le système social. Le discours permet de cerner comment un événement ponctuel bouscule le jeu des protagonistes et d'identifier quels types de tensions et conflits se manifestent le plus à l'égard des cours d'eau. De même est-il susceptible d'exprimer comment l'inquiétude liée à la perte annoncée d'écosystèmes dont la valeur écologique a été reconnue incite à développer une stratégie de protection ou de restauration d'habitats tels que les annexes fluviales.

Le discours fluvial connaît des variations spatiales et temporelles. L'analyse diachronique des écrits permet de repérer les évolutions d'un système autour des notions de rupture ou de tendance. Le temps de l'aménagement des cours d'eau par la construction de voies navigables, par les équipements hydroélectriques, par le développement des activités industrielles (notamment chimiques et nucléaires) ou encore par les ouvrages de protection fait place à une vision renouvelée des milieux aquatiques. La désignation des eaux courantes a évolué entre la loi sur le régime des eaux de 1898 et la loi sur l'eau et les milieux aquatiques (LEMA) de 2006. Le vocable de cours d'eau domaniaux s'est substitué à ceux de fleuves et rivières navigables ou flottables; les masses d'eau se sont affirmées avec la directive cadre européenne sur l'eau de 2000. Et même lorsque le mot ne change pas formellement, les sens qu'il charrie avec lui peuvent diverger. Tournier (1993) a rappelé en effet que Bakhtine distinguait trois éléments dans toute unité linguistique : (a) une signification qui désigne la permanence sémiotique d'un mot, c'est-à-dire ce qui ne change pas d'un emploi à l'autre, (b) un sens qui se rapporte à l'hétérogénité sémantique d'un mot, soit ce qui varie selon ses emplois, d'une situation d'énonciation à l'autre, et (c) la valeur d'accentuation (connotation positive ou négative) qui procède aussi de la situation. Or l'eau véhicule des enjeux dont la hiérarchie n'est pas fixée. Une étude des productions écrites doit ainsi être propice au questionnement de la prise de conscience environnementale dans les dispositifs de gestion : est-elle progressive ou bien est-elle reliée à des événements plus locaux, comme les aléas qui précipitent le processus de régulation ? Le discours peut aussi varier dans l'espace selon les héritages et les enjeux locaux. Peut-on cerner des attitudes environnementales à l'égard des eaux courantes à l'échelle du continuum fluvial ou bien faut-il raisonner à des échelles plus fines?

\section{Questionnements méthodologiques}

Concevant le discours comme une forme de pratique sociale, Fairclough (1995) invite à l'étudier selon une triple approche micro-analytique des textes (en décrivant leurs propriétés linguistiques), méso-analytique de la pratique discursive (en interprétant les relations qu'entretiennent les processus de production, distribution et consommation avec les textes) et macro-analytique des pratiques sociales (en expliquant dans quelle mesure le discours est 
socialement constitué et comment il contribue aussi à le (re)produire). Bien qu'elle ne soit pas exclusive des deux autres, la première entrée, centrée sur les textes, est privilégiée ici. A l'heure de la numérisation, les innovations technologiques sont telles que tout le processus de collecte, de traitement et d'interprétation des textes connaît des mutations profondes. Non seulement la quantité de matériel disponible s'accroît dans des proportions sans précédent, mais l'enrichissement permanent de l'offre logicielle ouvre des perspectives d'analyses stimulantes. Les discours portés à l'égard d'un objet de nature tel que les cours d'eau se prêtent particulièrement bien à la mise en œuvre des techniques dites d'analyse de contenu et d'analyse de données textuelles, en complément de méthodologies plus qualitatives. Les applications visent notamment à enrichir la connaissance et à questionner la complémentarité entre analyse de contenu et analyse de données textuelles.

L'analyse de contenu est apparue au début du XXe siècle pour classer les contenus de presse. Berelson (1952) l'a définie comme "une technique de recherche pour la description objective, systématique et quantitative du contenu manifeste de la communication" (p. 18). Bien que cette méthode se prête volontiers à une approche plus exploratoire d'un corpus constitué, le chercheur gagne à formuler des hypothèses explicites pour élaborer une grille de catégories cohérentes et pertinentes (Negura, 2006). Les critiques les plus vives portent précisément sur cette catégorisation, notamment lorsqu'elle requiert que l'analyste repère des catégories sémantiques (c'est-à-dire des thèmes) qui n'ont pas d'unité formelle objectivement repérable (Comby et al., 2012). Viennent ensuite l'étape du codage et du comptage d'unités d'analyse, et enfin celle de l'interprétation (Bardin, 1977; Robert et Bouillaguet, 2007). Ainsi cette technique permet-elle l'analyse des données textuelles qualitatives sous la forme de données quantitatives (Hayward et Osborne, 1973) : si la catégorisation et le codage des textes relèvent d'un processus qualitatif, le décompte de ces catégories permet des analyses statistiques (Boholm, 2009). En toute rigueur l'analyse de contenu s'en tient au contenu manifeste, mais l'interprétation de ses résultats donne accès au contenu latent, les variations de contenu permettant de faire des inférences.

En réaction au système des catégories définies a priori (inhérent à l'analyse de contenu), l'analyse de données textuelles s'est d'abord efforcée de repousser tout travail d'interprétation le plus tard possible (Lebart et Salem, 1988). Les logiciels de lexicométrie et de textométrie accompagnent désormais le chercheur tout au long de son effort de lecture, d'exploration, d'analyse et d'interprétation en lui proposant des outils divers: manipulation du corpus (aménagement de sous-corpus ou d'une partition), production de lexiques alphabétiques et hiérarchiques, création de requêtes sophistiquées qui débouchent sur un concordancier ou bien des index, analyses statistiques descriptives, calcul des spécificités et des cooccurrences, confection de tables lexicales propices aux analyses multivariées (analyse factorielle des correspondances et classification hiérarchique), ou encore des visualisations graphiques de plus en plus convaincantes. Les offres les plus récentes bousculent les frontières traditionnelles entre analyses qualitative et quantitative ou entre analyse de contenu et analyse de données textuelles : elles intègrent des modalités d'import susceptibles d'inciter l'opérateur à coder le texte au préalable ou bien à recueillir des métadonnées utiles lors de l'analyse, et permettent le retour au texte intégral à tout moment. Les textes du corpus peuvent donc être étudiés pour eux-mêmes mais aussi au regard des caractéristiques de leurs producteurs et consommateurs, ce qui facilite le test d'hypothèses concernant d'éventuelles variations spatiales, temporelles et socioculturelles du discours (Kah, 2000 ; Amalric, 2005 ; Germes et al., 2010 ; Germaine, 2011 ; Brennetot et al., 2013).

Dans les années 1970, réfutant un certain positivisme, des scientifiques ont dénigré une recherche fondée sur la mesure et les statistiques au profit d'une recherche qualitative qui se doit de retranscrire le monde réel vécu par les acteurs (Paillé, 2006). Ce travail au plus près des sources ne dénature pas le texte. Il se prête plus facilement aux ajustements des 
hypothèses initiales. L'originalité de ces méthodes peut procéder d'une lecture flottante, à la recherche de nouveautés et assumant la subjectivité de l'opérateur. Ces analyses reposent souvent sur un lien immédiat entre le texte et son commentaire.

Avec différentes méthodes, les chercheurs issus des SHS analysent leurs corpus pour explorer les pratiques et le penser/savoir des experts, des gestionnaires, des acteurs, des usagers et des riverains. Dans ce numéro de Géocarrefour, les cinq contributeurs - géographes, juriste et sociologue - adoptent des approches quantitatives et qualitatives, et effectuent bien souvent des navettes entre les deux, pour tirer le meilleur de documents à caractère verbal dont la nature se montre diverse, tantôt de source orale avec les campagnes d'entretiens directifs tantôt de source écrite avec les articles de presse, les documents historiques ou les textes juridiques. L'entrée par les discours permet de faire le point sur la complémentarité des méthodes pour collecter le dit et l'écrit, prétraiter leurs corpus, les analyser et les interpréter.

L'article de Marylise Cottet, géographe, traite du discours tenu à l'égard des bras morts sur la base d'une synthèse des acquis de la littérature scientifique et de transcriptions d'entretiens semi-directifs. Plus particulièrement, au moyen d'une analyse de contenu appliqué à ce dernier corpus, elle étudie les connaissances et les représentations liées à la structure, au fonctionnement et à l'évolution des annexes hydrauliques. Ses résultats identifient des points communs et des différences entre les changements paysagers, les facteurs explicatifs et les jugements de valeur qu'expriment les experts, les élus locaux et les usagers des cours d'eau. Aussi l'auteure discute-t-elle le rôle de l'observation, de l'expérience ou de la mémoire dans l'élaboration de la connaissance des individus; ce qui l'amène à préciser qu'une stratégie d'éducation à l'environnement et une réflexion sur les instances de concertation favorisent le succès des projets de restauration écologique.

Emeline Comby, géographe, explore un corpus d'articles publiés par la presse quotidienne régionale qui traitent de la reconquête des berges du Rhône et de la Saône dans l'agglomération lyonnaise entre 2003 et 2010. Elle met en œuvre une démarche contrastive à l'égard de cinq projets urbains pour questionner leurs dimensions spatiales et temporelles ainsi que les systèmes d'acteurs qui y sont liés, en recourant non seulement à l'analyse de contenu et à la textométrie, mais aussi à une approche davantage qualitative. Les résultats soulignent combien les discours tenus sur la reconquête des fronts d'eau urbains témoignent tout à la fois de la compétition interurbaine à laquelle les différentes métropoles se livrent pour renforcer leur attractivité et de l'identité intra-urbaine que les travaux contribuent à affermir.

Dans son étude sociologique, Christine Labeur interroge le rôle de la mémoire des catastrophes dans les pratiques riveraines à l'occasion d'une nouvelle crise. La composition de son corpus est originale puisque ce dernier réunit des discours écrits et oraux, à savoir deux textes liés aux débordements de 1755 et 1840, et des entretiens sur l'inondation de 2003. Suite à une analyse de contenu et à une analyse lexicométrique, les résultats montrent notamment que l'attitude tenue à l'égard d'une crise varie selon que la mémoire d'une catastrophe passée provient de l'expérience personnelle, a été communiquée par un proche ou bien relève d'une connaissance générale. De plus, les inondations (ré)activent des liens de solidarités que le discours permet d'appréhender.

Des géographes anglophones et francophones s'efforçant de dresser des passerelles entre le droit et la géographie (Blomley, 1989 et 1994 ; Forest, 2009), l'article d'Aude Farinetti, juriste, est plein d'actualité. L'auteure appréhende les mots comme des révélateurs de l'interface hommes-environnement: elle replace le texte dans son contexte de production, questionnant ainsi ce qui unit le droit et la science. En effet, au cours de l'époque contemporaine, le dispositif juridique français a enregistré des mutations. En particulier, à l'intérêt sectoriel porté à la satisfaction et à la pérennisation de quelques pratiques s'est 
substituée une approche davantage écologique qui a profité de l'exploration du concept d'hydrosystème fluvial dans les sciences environnementales.

C'est avec beaucoup de plaisir que nous avons adjoint à ce numéro un texte de Jacques Bethemont. Ancien directeur de la Revue de géographie de Lyon, il a participé à sa modernisation et reste un contributeur actif en proposant des articles et des comptes rendus d'ouvrages. Il est également un géographe des fleuves, et du Rhône en particulier, dont la production a été reconnue dans un ouvrage récent (Daudel, 2012). En novembre 2012, la médaille d'argent de la Société de Géographie lui a été décernée pour l'ensemble de son œuvre. Son article qui emprunte aux sources les plus diverses clôt ce numéro dans une langue qui mêle la poésie et la science avec bonheur.

Enfin, clôturant ce numéro, un article en varia de Jean-Baptiste Grison présente les mutations que connaissent les très petites communes françaises en insistant sur la diversité des situations. Si sa thématique diffère sensiblement de celle du dossier, cet article procède d'une enquête par courrier qui a été diffusée auprès des maires de plus de 1600 communes. Du discours a donc été provoqué puis analysé, même s'il n'a pas le même statut que dans les articles précédents. Au terme de la lecture du numéro, on pourra s'interroger sur le très fréquent recours au discours qui est fait dans le champ des SHS et l'inégal besoin de légitimation de la méthode selon la nature des sources traitées. Si la consultation des acteurs va presque de soi en géographie, et depuis longtemps, d'autres sources suscitent davantage de réticences et invitent à la prudence.

\section{Remerciements}

Ce numéro de Géocarrefour a bénéficié d'un soutien financier du projet intitulé "Encrer le fleuve Rhône. Le discours de la rupture au prisme des sciences humaines et sociales" et rédigé en réponse à l'appel à projet de recherche (APR) 2011 du Réseau des Observatoires HommesMilieux (OHM). L'Observatoire Hommes/Milieux de la Vallée du Rhône (OHM-VR), une plate-forme fédérative de recherche interdisciplinaire dont l'objectif principal consiste à questionner l'évolution contemporaine du fleuve et de ses sociétés riveraines, a également contribué. Ces travaux ont été prolongés en 2012-2013 au moyen d'un projet dont le titre souligne la filiation avec le précédent : "Mieux comprendre les discours de crise sur le fleuve Rhône : extrêmes hydrologiques et plantes invasives", financé au sein de l'Accord Cadre Zone Atelier Bassin du Rhône (ZABR)-Agence de l'eau Rhône Méditerranée Corse (RMC).

\section{Bibliographie}

AJZEN I., 2005, Attitudes, Personality and Behavior, Maidenhead, Open University Press, $192 \mathrm{p}$.

ALLARD P., 2005, La presse et les inondations dans la région du bas Rhône en 1840 et 1856, in FAVIER R. et GRANET-ABISSET A.-M. (dir.), Récits et représentations des catastrophes depuis l'Antiquité, Grenoble, Publications de la MSH-Alpes, p. 73-92.

AMALRIC M., 2005. Les zones humides, appropriations et représentations. L'exemple du Nord-Pas de Calais, Thèse de géographie, Lille 1, 468 p.

BARDIN L., 1977, L'analyse de contenu, Paris, Presses Universitaires de France, 233 p.

BERELSON B., 1952, Content Analysis in Communication Research, Glencoe, The Free Press, $220 \mathrm{p}$.

BERQUE A., 1990, Médiance, de milieux en paysages, Montpellier, Reclus, 162 p.

BERQUE A., 1987, Milieu et motivation paysagère, L'Espace géographique, vol. 16, $\mathrm{n}^{\circ} 4$, p. 241-250. 
BERQUE A., 1985, Milieu, trajet de paysage et déterminisme géographique, L'Espace géographique, vol. 14, n² 2, p. 99-104.

BERQUE A., 1984, Paysage-empreinte, paysage-matrice : éléments de problématique pour une géographie culturelle, L’Espace géographique, vol. 13, n 1, p. 33-36.

BLOMLEY N., 1994, Law, space and the geographies of power, New York, Guilford Press, 259 p.

BLOMLEY N., 1989, Text and context: Rethinking the law-space nexus, Progress in Human Geography, vol. 13, n 4, p. 512-534.

BOHOLM M., 2009, Risk and Casualty in Newspaper Reporting, Risk Analysis, vol. 29, $\mathrm{n}^{\circ} 11$, p. 1566-1577.

BRENNETOT B., EMSELLEM K., GUERIN-PACE F. et GARNIER B., 2013, Dire l'Europe à travers le monde, Cybergeo, URL : http://cybergeo.revues.org/25684

BRUNET R., 1974, Espace, perception et comportement, L'Espace géographique, vol. 3, $\mathrm{n}^{\circ}$ 3, p. 189-204.

CARON A. et TORRE A., 2006, Vers une analyse des dimensions négatives de la proximité. Les conflits d'usage et de voisinage dans les espaces naturels et ruraux, Développement Durable et Territoires, URL : http://developpementdurable.revues.org/2641

COMBY E., LE LAY Y.-F. et PIEGAY H., 2012, La presse, une source pour l'étude spatiale et temporelle des attitudes. Potentialités et outils d'analyses des discours sur les crues, Dixièmes Rencontres de Théo Quant, URL : http://thema.univfcomte.fr/theoq/pdf/2011/TQ2011\%20ARTICLE\%205.pdf

DAUDEL C., 2012, Jacques Bethemont. Géographe des fleuves, Paris, L'Harmattan, 350 p.

DEPRAZ S., 2005, Le concept d' «Akzeptanz » et son utilité en géographie sociale. Exemple de l'acceptation locale des parcs nationaux allemands, L'Espace géographique, vol. $34, \mathrm{n}^{\circ} 1$, p. 1-16.

DIEL P., 1968, La peur et l'angoisse. Phénomène central de la vie et de son évolution, Paris, Payot, 214 p.

DURAND D., 2010, La systémique, Paris, PUF, 127 p.

FAIRCLOUGH N., 1995, Critical discourse analysis: The critical study of language, London, Longman, 265 p.

FESTINGER L., 1957, A theory of cognitive dissonance, Evanston, Row Peterson, 291 p.

FLAMENT C., 1994, Structure, dynamique et transformation des représentations sociales, in ABRIC J.-C. (dir.), Pratiques sociales et représentations, Paris, PUF, p. 37-58.

FOREST P. (dir.), 2009, Géographie du droit. Epistémologie, développement et perspectives, Québec, Les Presses de l'Université de Laval, 286 p.

FRISSELL C.A., LISS W.J., WARREN C.E. et HURLEY M.D., 1986, A hierarchical framework for stream habitat classification: Viewing streams in a watershed context, Environmental Management, vol. 10, n 2, p. 199-214.

GERMAINE M.-A., 2011, Apport de l'analyse de discours pour renseigner les représentations paysagères et les demandes d'environnement. Les vallées du nord-ouest de la France, Annales de géographie, ${ }^{\circ}$ 682, p. 629-650.

GERMES M., BRAILICH A., SCHIRMEL H., GLASZE G. et PÜTZ R., 2010, Les grands ensembles de banlieue comme menaces urbaines ? Discours comparés - Allemagne, France, Pologne, Annales de géographie, $\mathrm{n}^{\circ}$ 675, p. 515-535.

GOLD J.R., 1980, An introduction to behavioural geography, Oxford, Oxford University Press, $290 \mathrm{p}$.

GOLLEDGE R.G. et STIMSON R.J., 1997, Spatial behavior. A geographic perspective, New York, The Guilford Press, 620 p.

GUERIN-PACE F. et COLLOMB P., 1998, Les contours du mot "environnement" : enseignements de la statistique textuelle, L'Espace géographique, nº 1, p. 41-52. 
HEIDER F., 1946, Attitudes and cognitive organization, Journal of Psychology, vol. 21, $\mathrm{n}^{\circ} 1$, p. 107-112.

HÖLDERLIN F., 2005, Euvre poétique complète, Paris, éditions de La Différence, 1007 p.

KAH E., 2000, Un problème de préservation de l'environnement en géographie : l'élimination des déchets ménagers et le comportement des usagers. L'exemple du consentement à payer, Thèse de géographie, Université Louis Pasteur - Strasbourg, 442 p.

KOESTLER A., 1979, Janus, Paris, Calmann-Lévy, 348 p.

LABORIT H., 1994, Les bases biologiques des comportements sociaux, Québec, Musée de la civilisation, $59 \mathrm{p}$.

LEBART L. et SALEM A., 1988, Analyse statistique des données textuelles. Questions ouvertes et lexicométrie, Paris, Dunod, 210 p.

LE BRETON D., 1995, Sociologie du risque, Paris, Presses Universitaires de France, 128 p.

MACLEAN P., 1990, Les trois cerveaux de l'homme, Paris, Robert Laffont, 367 p.

MORIN E., 2000, Les sept savoirs nécessaires à l'éducation du futur, Paris, Seuil, 129 p.

MORIN E., 1994, La complexité humaine, Paris, Flammarion, 380 p.

MOODIE D.W., 1971, Content Analysis: A Method for Historical Geography, Area, vol. 3, n 3, p. 146-149.

NEGURA L., 2006, L'analyse de contenu dans l'étude des représentations sociales, SociologieS. Théories et recherches, URL : http://sociologies.revues.org/index993.html

PAILLE P. (dir.), 2006, La méthodologie qualitative. Postures de recherche et travail de terrain, Paris, Armand Colin, 240 p.

PIGEON P., 2005, Géographie critique des risques, Paris, Economica, 217 p.

ROBERT A.D. et BOUILLAGUET A., 2007, L'analyse de contenu, Paris, Presses Universitaires de France, 127 p.

ROSENBERG M.J., HOVLAND C.I., MCGUIRE W.J., ABELSON R.P., BREHM J.W., 1960, Attitude organization and change. An analysis of consistency among attitude components, New Haven, Yale University Press, 239 p.

ROUSSIAU N. et BONARDI C., 2001, Les représentations sociales. Etats des lieux et perspectives, Sprimont, Mardaga, $250 \mathrm{p}$.

SCHOENEICH P. et BUSSET-HENCHOZ M.-C., 1998, La dissonance cognitive : facteur explicatif de l'accoutumance au risque, Revue de Géographie Alpine, vol. 86, n² 2, p. 53-62.

THOURET J.-C. et D'ERCOLE R., 1996, Vulnérabilité aux risques naturels en milieu urbain : effets, facteurs et réponses sociales, Cahiers des Sciences Humaines, vol. 32, n 2, p. 407-422. TOURNIER M., 1993, Lexicometria, Lisbonne, Universidade Aberta, 127 p.

TOURNIER M., 1981, La réduction : principe de la lexicométrie politique, In Computational lexicology and lexicography. Special issue dedicated to Bernard Quemada, Pisa, Giardini Editori, p. 277-292.

TOURNIER M., 1980, D'où viennent les fréquences de vocabulaire ? La lexicométrie et ses modèles, Mots, n 1, p. 189-209.

TRIANDIS H.C., 1971, Attitude and attitude change, New York, Wiley, 232 p.

VALLERAND R.J. (dir.), 2006, Les fondements de la psychologie sociale, Boucherville, Gaëtan Morin éditeur, 741 p.

VANNOTE R.L., MINSHALL G.W., CUMMINS K.W., SEDELL J.R., CUSHING C.E., 1980, The River Continuum Concept, Canadian Journal of Fisheries and Aquatic Sciences, vol. 37, n 7, p. 130-137.

VEYRET Y. et REGHEZZA M., 2006, Vulnérabilité et risques. L’approche récente de la vulnérabilité, Annales des Mines. Responsabilité \& environnement, n 43, p. 9-13.

VICARD F., AZNAR O., BRETIERE G. et HERVIOU S., 2005, Une analyse des services environnementaux produits dans un département français, VertigO, vol. 6, n 3, 8 p., URL : http://vertigo.revues.org/3703. 
WARD J.V., 1989, The four-dimensional nature of lotic ecosystems, Journal of the North American Benthological Society, vol. 8, n 1, p. 2-8.

\section{Table des figures}

Figure 1. Approche anthroposystémique des relations hommes-environnement.

Figure 2. Dissonance cognitive et attitudes à l'égard de l'environnement.

Figure 3. Le risque, l'anticipation anxiogène d'une crise.

Figure 4. Quelques modalités de l'action paysagère ou environnementale.

Figure 5. Trois grands types de réduction (Tournier, 1980, 1981 et 1993). 
Figure 1. Approche anthroposystémique des relations hommes-environnement.

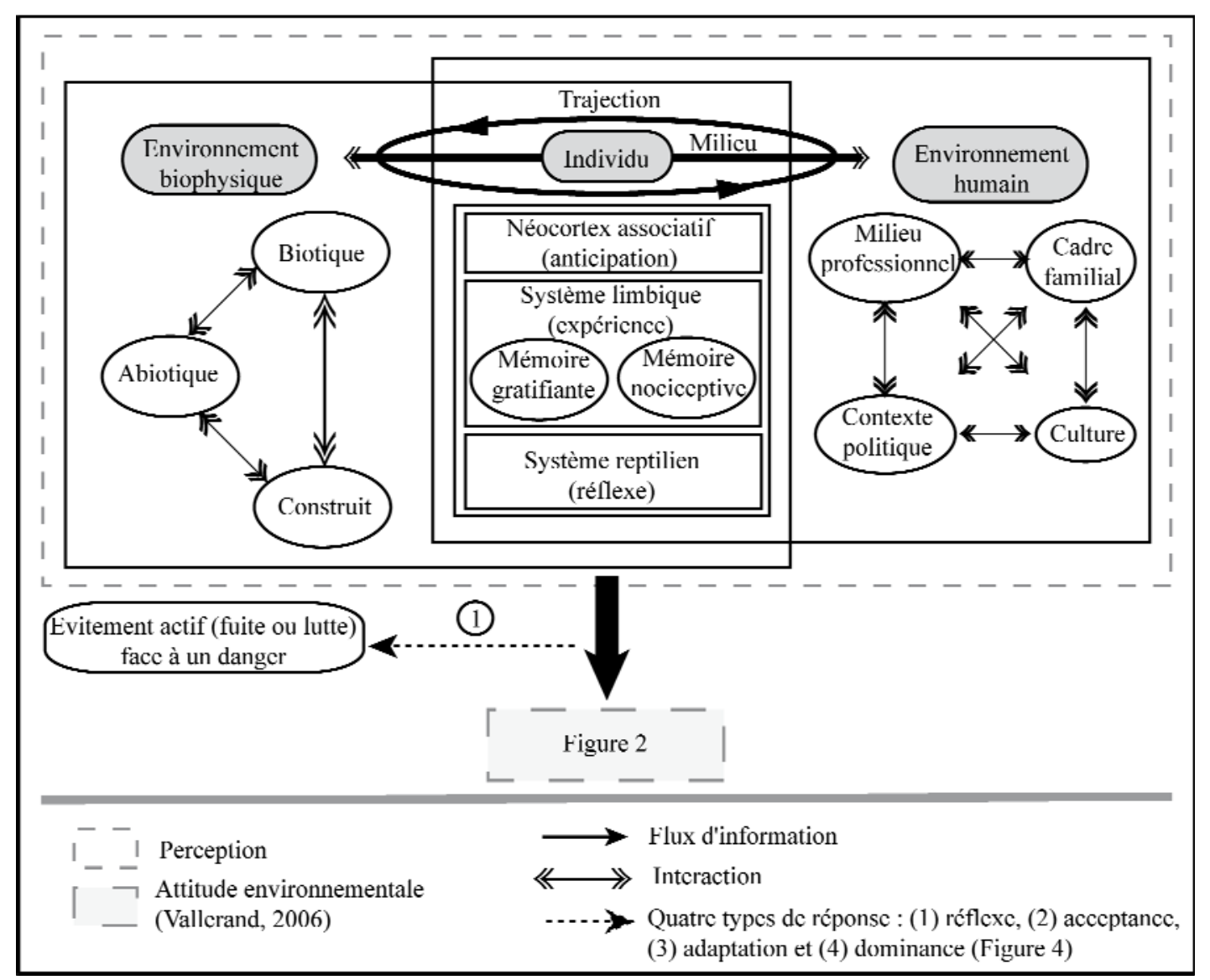


Figure 2. Dissonance cognitive et attitudes à l'égard de l'environnement.

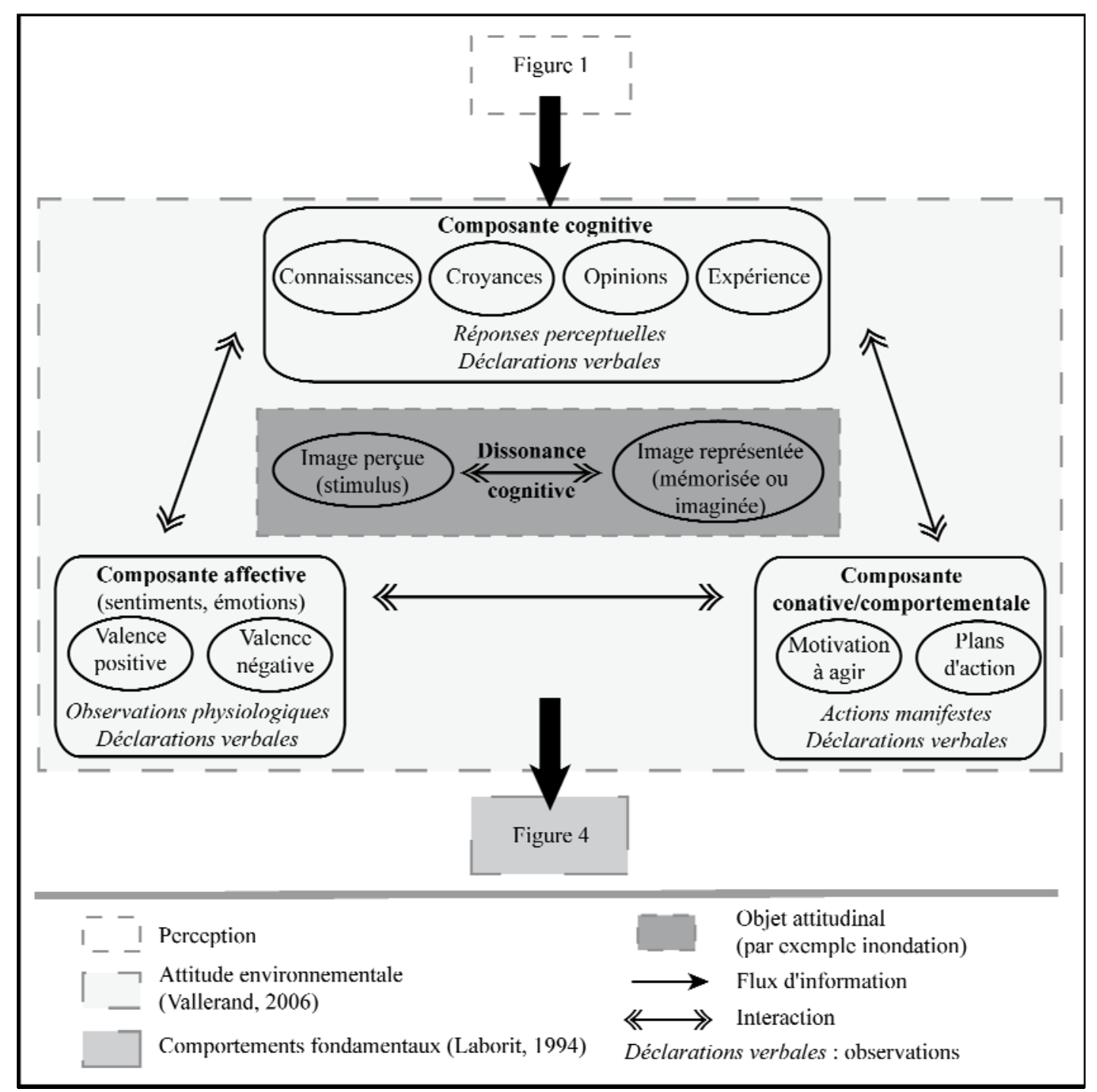


Figure 3. Le risque, l'anticipation anxiogène d'une crise.

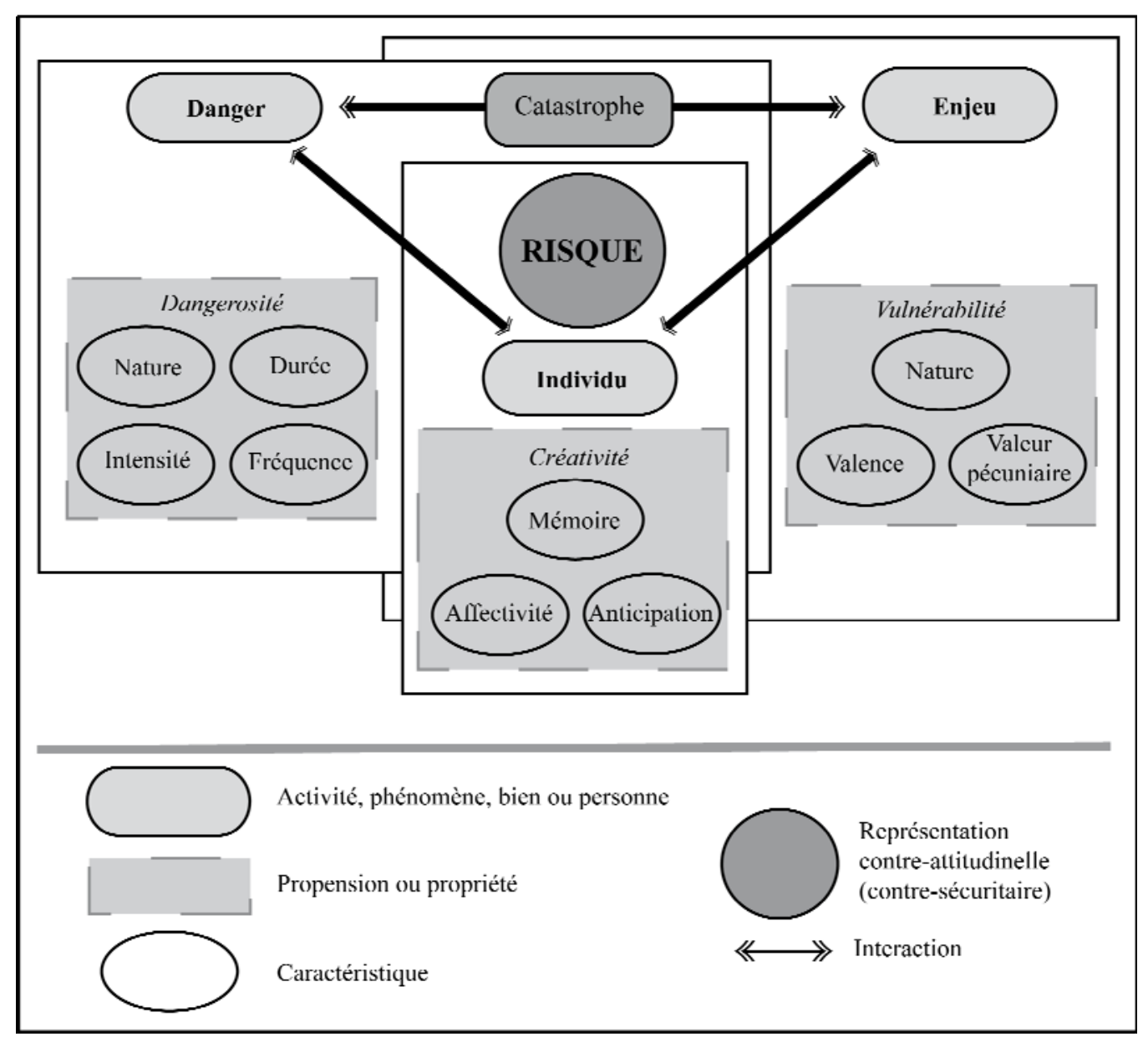


Figure 4. Quelques modalités de l'action paysagère ou environnementale.

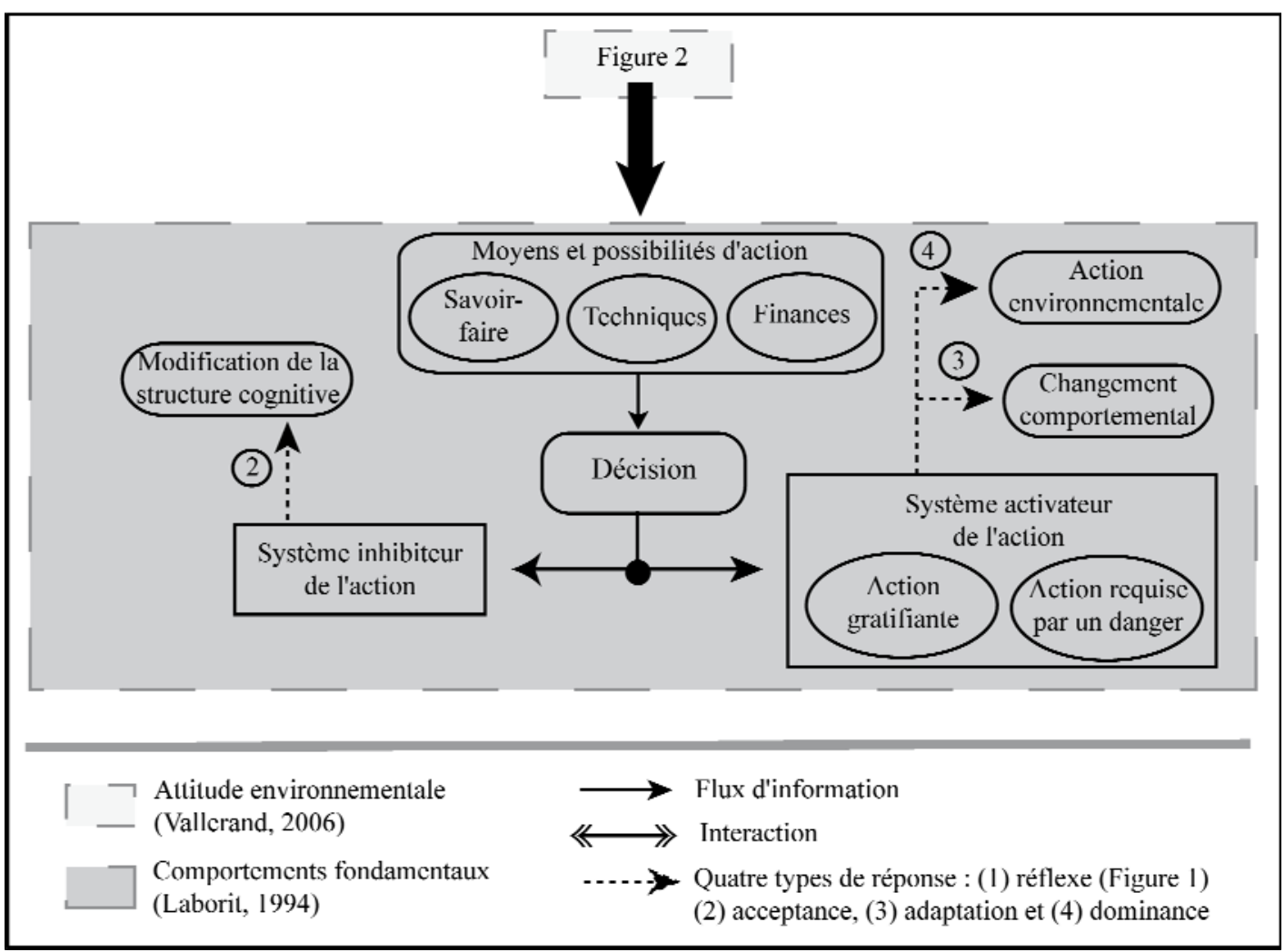


Figure 5. Trois grands types de réduction (d'après Tournier, 1980, 1981 et 1993).
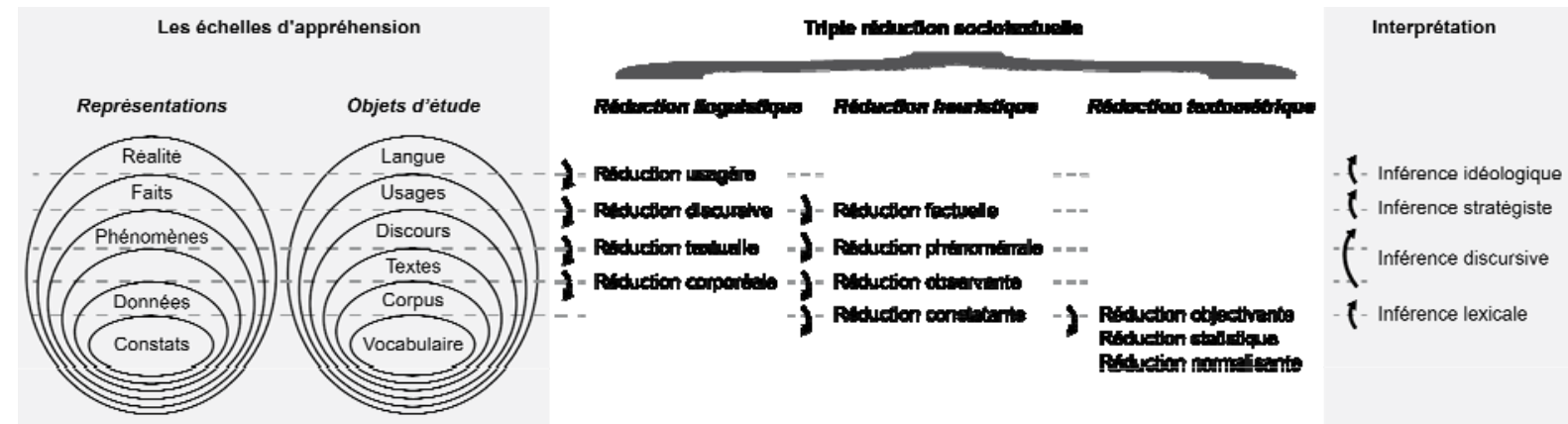